\title{
Etude de la viabilité d'un modèle parabolique de Berkhoff appliqué aux abords de l'embouchure de l'Adour.
}

\author{
Christophe Brière ${ }^{(1)}$, Stéphane Abadie ${ }^{(2)}$ \\ (1): Doctorant LaSAGeC - Génie côtier, Université de Pau et des \\ Pays de l'Adour, I.S.A.B.T.P., 1 Allée du Parc Montaury, 64600 \\ Anglet, France, E-mail: christophe.briere@univ-pau.fr, Tel: \\ 05.59.57.70.42 \\ (2): Maître de Conférences LaSAGeC - Génie côtier, Université de \\ Pau et des Pays de l'Adour, I.S.A.B.T.P., 1 Allée du Parc Montaury, \\ 64600 Anglet, France, E-mail: stephane.abadie@univ-pau.fr, Tel: \\ 05.59.57.70.48
}

\section{Résumé :}

Le régime extrême des houles agissant sur le littoral angloy provoque une érosion de l'estran et des petits fonds relativement importante. Confrontée au problème, la communauté d'agglomérations Bayonne-Anglet-Biarritz a confié au Laboratoire des Sciences Appliquées au Génie Civil (LaSAGeC) une étude complète à long terme de cette problématique locale dont une partie vise au développement d'un modèle numérique couplé houle/courant/sédiment. L'objet de cette communication est la présentation des premiers résultats concernant la modélisation de la houle par utilisation d'un modèle simplifié résolvant l'équation de Berkhoff sous sa forme parabolique (REF-DIF). La particularité du site (présence de digues et épis, embouchure de l'Adour) modifie la propagation des ondes de manière complexe et certaines limites du modèle REF-DIF, concernant en particulier le traitement des digues et épis, sont mises en évidence.

\section{Abstact :}

The strong wave climate acting along Anglet coastline is responsible for an erosion of beaches for decades. The $\mathrm{LaSaGeC}$ is in charge of a global study of this problem. One aspect of this investigation consists in developing a morphodynamical model. This communication aims at presenting the first results of a simplified wave propagation model based on the parabolic approximation of the Berkhoff equation (REF-DIF). Characteristic features of the study area (jetties, breakwater, Adour river mouth) alter significantly the wave propagation and shows some limits of applicability of the model especially concerning the treatment of the seawalls.

\section{Introduction}

La côte aquitaine et plus particulièrement la côte basque est soumise à des régimes de houles importants et les mouvements sédimentaires induits sont tels que le littoral subit une érosion importante depuis quelques décennies. La 
Communauté d'Agglomérations Bayonne-Anglet-Biarritz a chargé le LaSAGeC de réaliser une étude de l'évolution du trait de côte angloy afin de mieux appréhender les différents phénomènes hydrosédimentaires présents. En particulier, il est, à terme, prévu de réaliser un modèle morphodynamique couplé dont les trois modules sont présentés en figure 1.

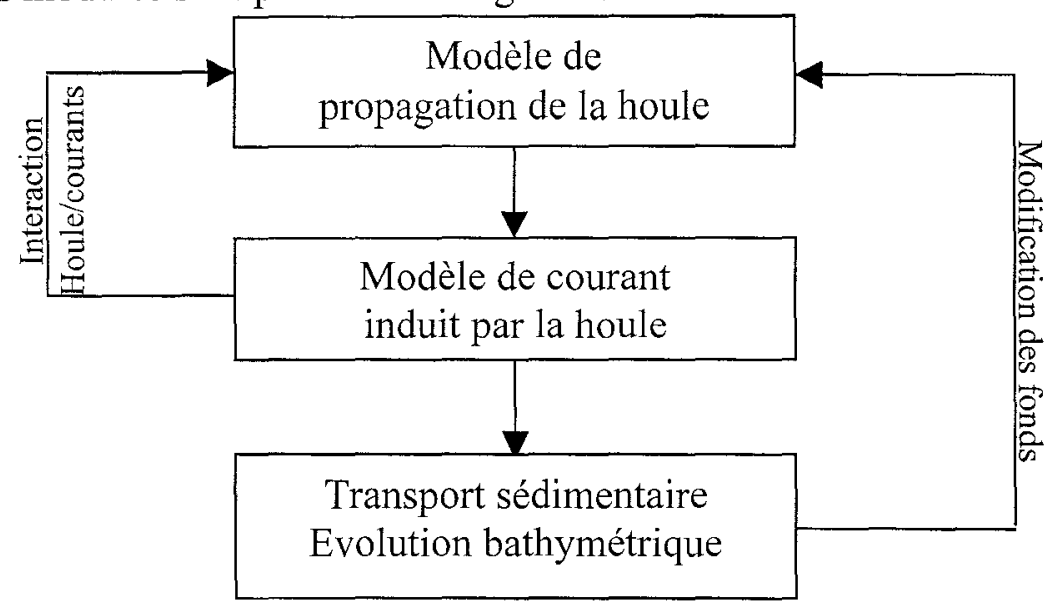

Fig. 1 : Modèle morphodynamique couplé.

Fig. 1 : Coupled morphodynamical model.

La modélisation est dite couplée dans le sens où chaque module influence directement le module qui suit, avec une boucle de retour du dernier module vers le premier. Ainsi, le champ de houle génère des courants moyens qui entraînent un déplacement de sédiments modifiant la bathymétrie et donc en retour la propagation de la houle.

Dans cet article, nous présentons les résultats concernant le premier module obtenus avec le modèle de propagation de houle REF-DIF [2], développé à l'Université de Delaware sur la base de l'équation de Berkhoff [3] ou équation «mild slope » sous sa forme parabolique [4]. Tout d'abord, une présentation du site d'étude ainsi que de la stratégie expérimentale d'acquisition des données de houle et des données bathymétriques est proposée. En second lieu, les différentes théories permettant d'estimer les paramètres de houle sont présentées. Une attention particulière est donnée à l'écriture de l'équation de Berkhoff sous sa forme parabolique. Par la suite, les premières limites d'utilisation du modèle pour le site d'Anglet sont mises en évidence et un retour à un cas test est réalisé en dernière partie afin de découpler les problèmes rencontrés. Une conclusion est enfin proposée quant à l'utilisation d'un modèle parabolique pour le site particulier d'Anglet.

\section{Description de la zone d'étude}

Les plages d'Anglet s'étendent sur un linéaire de 4,5 kilomètres orienté Sud-Sud-Ouest Nord-Nord-Est, entre l'embouchure de L'Adour et le Cap SaintMartin et constituent une province sédimentaire quasi-autonome [5]. La frontière profonde du domaine d'étude a été assimilée à la profondeur de fermeture calculée d'après la formule de Hallermeier [6], soit $\mathrm{h}=12,5 \mathrm{~m}$. 
La zone d'étude se situe, en coordonnées Lambert III, dans une surface définie entre $140250 \mathrm{~m} .<\mathrm{x}<147000 \mathrm{~m}$. et $284500 \mathrm{~m}$. $<\mathrm{y}<289250 \mathrm{~m}$. Par un changement de repère, rotation de 1,047 radians avec pour origine le point de coordonnées Lambert III (140100, 285800), l'axe Ox est sensiblement parallèle au littoral. L'obtention d'une bathymétrie interpolée est réalisée à l'aide du logiciel «Surfer 7» de Golden Software à partir de données brutes acquises par un système DGPS et sondeur acoustique de précision centimétrique. La figure 2 montre la bathymétrie mesurée le 16 Novembre 2001.

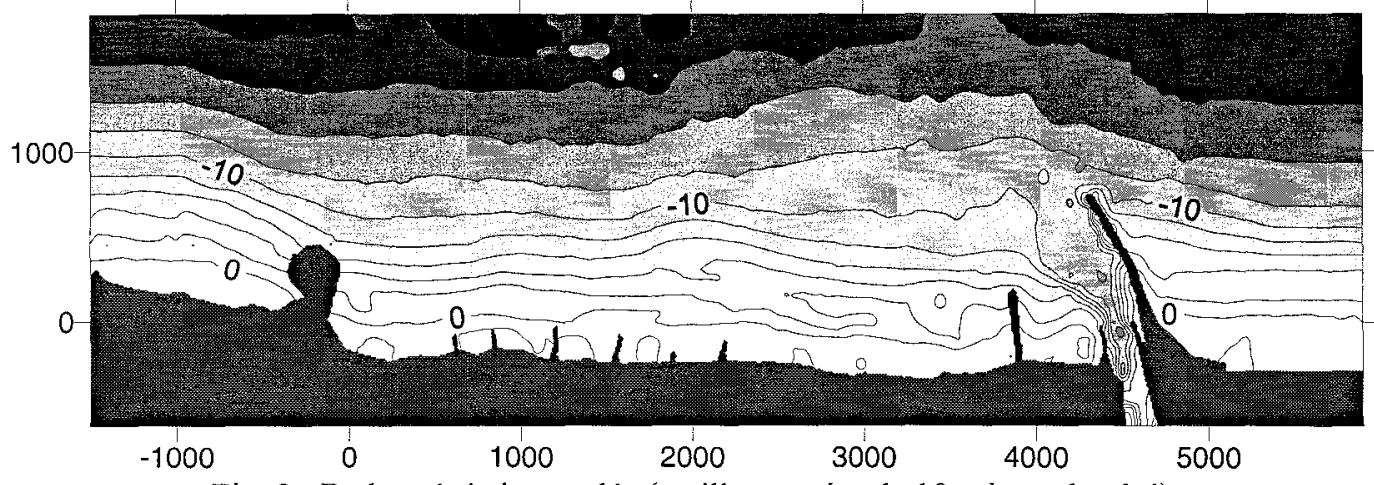

Fig. 2 : Bathymétrie interpolée (mailles carrées de 10 mètres de côté).

Fig. 2 : Interpolated bathymetry (10 meters mesh cell size).

Début 2002, le LaSAGeC va installer au large d'Anglet par $50 \mathrm{~m}$. de fond une bouée de houle directionnelle de marque TRIAXYS avec la collaboration du Centre d'Etudes Techniques Maritimes Et Fluviales (CETMEF). Elle mesurera les données de houle au large nécessaires en entrée du modèle de propagation.

Pour cette communication, on utilise des données relatives aux régimes des houles au large d'Anglet mesurées lors d'études antérieures. Une synthèse des documents [7] du Laboratoire Central d'Hydraulique de France et des données CANDHIS du CETMEF, rassemblant les mesures d'états de mer réalisées par différents organismes, permet de caractériser le type de houles à utiliser en entrée du modèle. Deux régimes distincts, dits « d'hiver » et "d'été », sont mis en évidence et les paramètres de houle représentatifs de ces régimes sont utilisés pour tester le modèle REF-DIF.

\section{Description du modèle de houle}

\subsection{Choix d'une méthodologie}

La théorie de la réfraction pure suppose que la hauteur de la houle sur une même crête varie lentement, il n'y a pas d'échange d'énergie entre les orthogonales. Cette approche est à la base des modèles de rayons qui permettent de calculer la propagation des lignes de crêtes.

Cependant, dans des zones d'étude semi-fermées, cette hypothèse n'est plus vérifiée : la réflexion et la diffraction interviennent et la variation de la hauteur n'a plus lieu uniquement selon la direction orthogonale à la crête mais dans le plan horizontal. L'équation «mild slope» incluant les effets de la diffraction, de la réfraction, de la réflexion, et la dissipation par déferlement, représente alors une alternative intéressante. Sa forme elliptique est bien adaptée 
aux systèmes portuaires dits fermés tandis que sa forme parabolique aux systèmes côtiers dits ouverts.

La propagation de la houle en faible profondeur étant un phénomène non linéaire, une autre approche consiste à considérer les équations de Boussinesq standards [8] étendues aux profondeurs variables [9]. Cependant, cette approche n'est pas très économique, en particulier pour des zones de grande emprise comme le site d'Anglet.

Les modèles spectraux tiennent également compte des phénomènes non linéaires mais leur utilisation semble plutôt appropriée aux applications de types océanographique et météorologique. Le modèle SWAN en est un exemple où la houle y est décrite à l'aide du spectre de densité d'action [10].

Compte tenu des caractéristiques de notre site d'étude qui peut être considéré comme semi-ouvert (emprise large, ouvrages limités excepté la digue Nord), l'utilisation de l'équation de Berkhoff sous sa forme parabolique semble de prime abord adaptée à la modélisation des phénomènes physiques présents.

\subsection{L'équation « mild slope » parabolique}

Si on se place dans le cadre de la théorie de la houle linéaire, on peut définir un potentiel des vitesses $\phi(x, y, z)$ qui vérifie l'équation de Laplace et les conditions limites au fond $(z=-h)$ et en surface $(z=0)$ suivantes :

$$
\begin{array}{ll}
\Delta \phi=0 & \text { pour }-h(x, y) \leq z \leq 0 \\
\frac{\partial^{2} \phi}{\partial t^{2}}+g \frac{\partial \phi}{\partial z}=0 & \text { pour } z=0 \\
\frac{\partial \phi}{\partial z}=-\frac{\partial \phi}{\partial x} \frac{\partial h}{\partial x}-\frac{\partial \phi}{\partial y} \frac{\partial h}{\partial y} & \text { pour } z=-h(x, y)
\end{array}
$$

On suppose que tout se passe comme si on avait, au voisinage de chaque point, une houle linéaire dont l'amplitude varierait dans le plan horizontal. Pour rendre le problème bidimensionnel, l'équation de Laplace est intégrée sur la verticale. On déduit, après prise en compte des conditions aux limites et de la relation de dispersion, l'équation de Berkhoff ou équation pour faibles pentes :

$$
\nabla\left(c c_{g} \nabla \Phi\right)+k^{2} c c_{g} \Phi=0
$$

où $\Phi(x, y)$ est le potentiel bidimensionnel des vitesses, $\mathrm{k}$ le nombre d'onde et $c$ et $c_{g}$ sont respectivement les vitesses de phase et de groupe de l'onde.

L'hypothèse principale sur laquelle repose la formulation de l'équation de Berkhoff est la considération d'une faible variation du potentiel des vitesses selon l'axe z par rapport à sa variation dans le plan $(\mathrm{x}, \mathrm{y})$ permettant de décomposer le potentiel $\phi(x, y, z)$ en produit de fonctions des variables verticale et horizontales. 
En posant $\varphi=\sqrt{c c_{g}} \Phi$, on montre que l'équation elliptique de Berkhoff peut s'écrire sous forme d'une équation d'Helmotz. En dissociant le champ transmis du champ réfléchi et en négligeant ce dernier, on déduit :

$$
\frac{\partial \varphi}{\partial x}=\left(i k-\frac{1}{2 k c c_{g}} \frac{\partial}{\partial x}\left(k c c_{g}\right)\right) \varphi+\frac{i}{2 k c c_{g}} \frac{\partial}{\partial y}\left(c c_{g} \frac{\partial \varphi}{\partial y}\right)
$$

Cette équation est appelée équation de Berkhoff parabolisée. Elle présente l'avantage de pouvoir être résolue ligne par ligne depuis le large jusqu'à la côte, ce qui limite le stockage en mémoire.

\section{Application au domaine d'étude.}

Le modèle REF-DIF nécessite au moins cinq nœuds par longueur d'onde. $\mathrm{Au}$ large d'Anglet, celle-ci est approximativement de cent mètres (profondeur de 20 mètres pour des périodes autour de 10 secondes). Une discrétisation par pas de 10 mètres vérifie ces conditions et a été adoptée pour les tests.

\subsection{Conditions limites latérales}

Pour obtenir la solution de l'équation différentielle parabolique considérée (Eq. 5), seules des conditions limites latérales et en amont sont nécessaires, la réflexion des ondes étant ignorée. Au travers de la limite latérale, on considère une transmission partielle de l'énergie de l'onde permettant un minimum de réflexion au sein du domaine. Il est cependant important de tester le modèle en considérant les limites latérales fermées (réflexion selon les bords) afin de visualiser l'influence de ces limites sur la zone d'étude et d'ajuster au mieux l'extension latérale du domaine à modéliser [2].

D'un point de vue numérique, le modèle parabolique est résolu par un schéma aux différences finies et le calcul des amplitudes est réalisé à l'aide d'une procédure implicite de Crank-Nicolson. Pour une ligne donnée, le schéma s'écrit sous la forme $a A_{i+1, j+1}+b A_{i+1, j}+c A_{i+1, j-1}=d A_{i, j+1}+e A_{i, j}+f A_{i, j-1}$, où a, b, c, d, e et $\mathrm{f}$ sont des termes réajustés pour chaque ligne en fonction des amplitudes préalablement calculées, et $\mathrm{i}$ et $\mathrm{j}$, respectivement, les indices des lignes parallèles à la côte et des colonnes. Un nœud a une influence directe sur les trois nœuds adjacents de la ligne suivante et les paramètres estimés en un nœud situé en limite latérale du domaine sont transmis suivant une oblique de $45^{\circ}$ à cette limite. Ainsi, le domaine doit être élargi de part et d'autre du site d'intérêt d'une distance équivalente à la longueur modélisée perpendiculairement à la côte.

Une étude menée en considérant les différents régimes de houle locaux montre cependant que l'influence des limites latérales est plus ou moins marquée selon l'importance de l'incidence et de l'amplitude de l'onde. Dans notre cas, une augmentation de $40 \%$ de la largeur perpendiculaire à la côte modélisée, de part et d'autre du Cap Saint Martin et de la digue Nord, est nécessaire au vu du climat de houle local. 


\subsection{Dissipations d'énergie}

Les dissipations d'énergie sont intégrées à l'équation pour faibles pentes à l'aide d'un facteur de dissipation $\sigma$ dépendant du phénomène considéré (frottements au fond et en surface ou déferlement). Cependant, la distance de propagation, pour les cas de simulation étudiés, est trop courte pour que les effets au fond et en surface puissent être significatifs numériquement.

\subsection{Problèmes numériques liés aux ouvrages}

La distribution des amplitudes au sein du domaine d'étude est ensuite calculée pour des conditions de houle représentatives du régime local, soit, entre autre, pour une onde d'incidence Ouest-Nord-Ouest, d'amplitude 1 mètre et de période 11 secondes. Le résultat pour ce cas de simulation est présenté en figure 3.

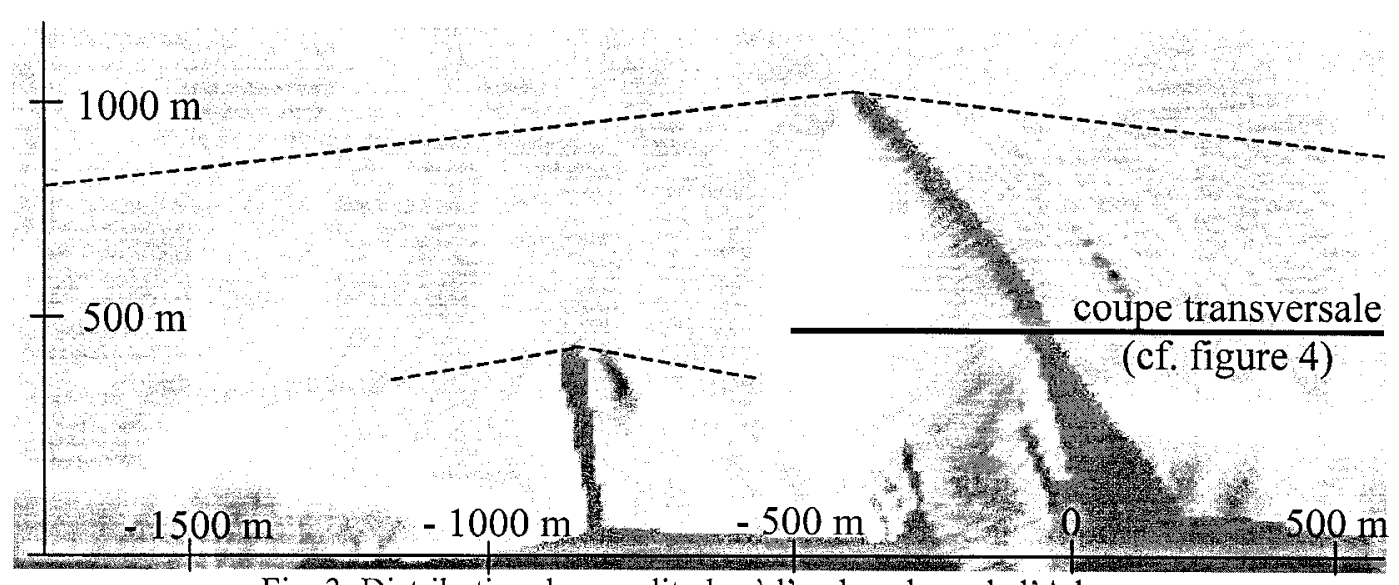

Fig. 3: Distribution des amplitudes à l'embouchure de l'Adour.

Fig. 3: Adour inlet wave height distribution.

La visualisation des résultats en figure 4 met en évidence des oscillations entre 1 et 3,5 mètres des hauteurs au nord de la digue principale (digue Nord).

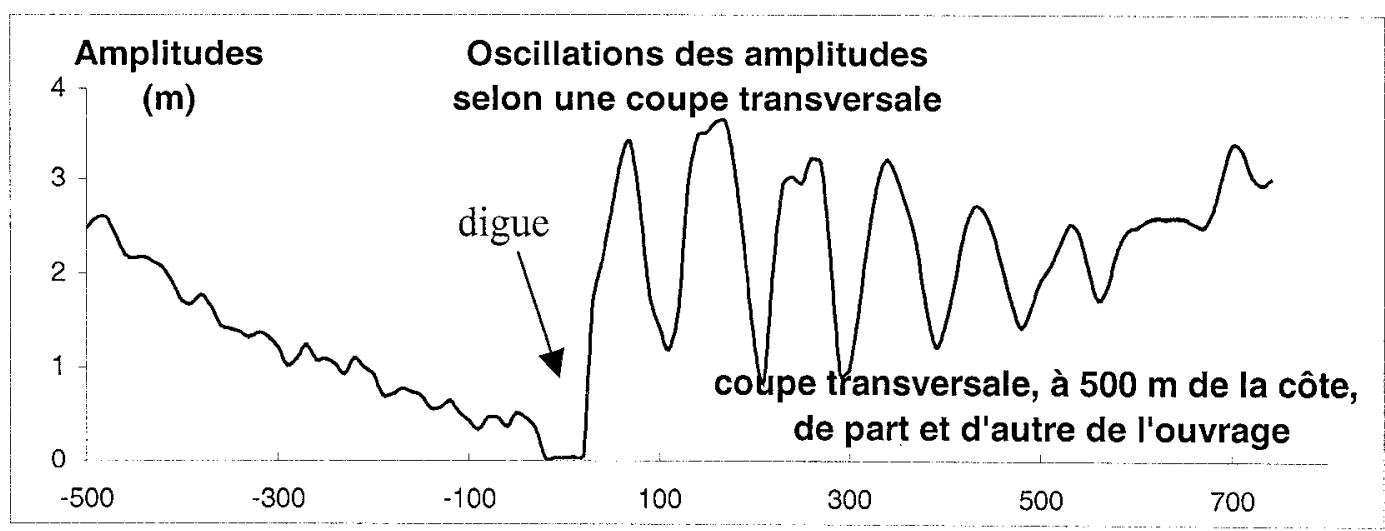

Fig. 4 : Distribution transversale des hauteurs.

Fig. 4 : long-shore heights distribution.

Le schéma numérique utilisé intègre mal les différences de bathymétrie devant les ouvrages. Les résultats obtenus par forte pente vont à l'encontre de 
l'hypothèse de faible pente et d'élimination des composantes réfléchies. Le modèle répercute, aux lignes suivantes les paramètres accidentés. Cette constatation se vérifie pour chacun des autres cas de simulation.

\section{Etude d'un cas d'ouvrage idéalisé}

Afin de mieux quantifier l'influence d'un ouvrage sur la distribution des amplitudes, la propagation d'une onde est étudiée dans un domaine (2000 m. X $2000 \mathrm{~m}$.) de pente 1:100 et comportant une digue perpendiculaire à la côte de 20 mètres de largueur et de 500 mètres de longueur. Le comportement des amplitudes est ensuite étudié en créant une plage de pente constante autour de l'ouvrage initial. Différents cas sont simulés pour des pentes 1:1, 1:2, 1:3, 1:4, $1: 8,1: 20,1: 25$ et 1:30, fonction de l'angle $\alpha$ (figure 5).

Les ouvrages en enrochements tels que ceux d'Anglet permettent en effet le déferlement de l'onde incidente le long de deux-ci et limitent la réflexion de la houle. Le but recherché est de créer un système de pente plus douce permettant de considérer une variation bathymétrique au sein du modèle et limitant les oscillations des amplitudes de part et d'autre de l'ouvrage.

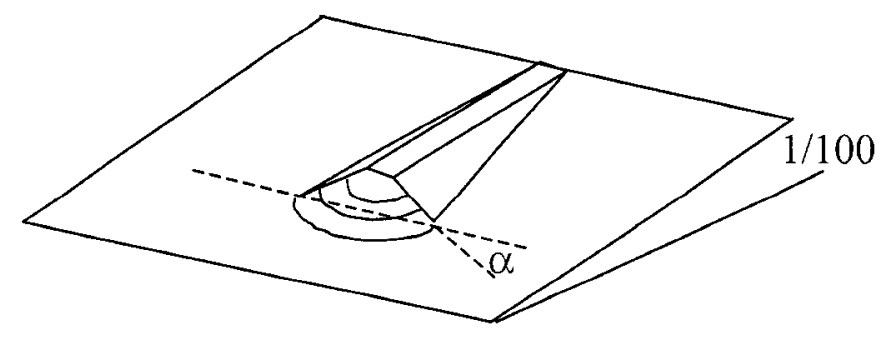

Fig. 5: Schéma descriptif du système étudié.

Fig. 5 : Description of the studied system.

Les tests ont été réalisés pour une onde d'incidence nulle, de hauteur 1 mètre et de période 8 secondes (figure 6 ) et pour une onde d'incidence $30^{\circ}$, de même hauteur et même période.

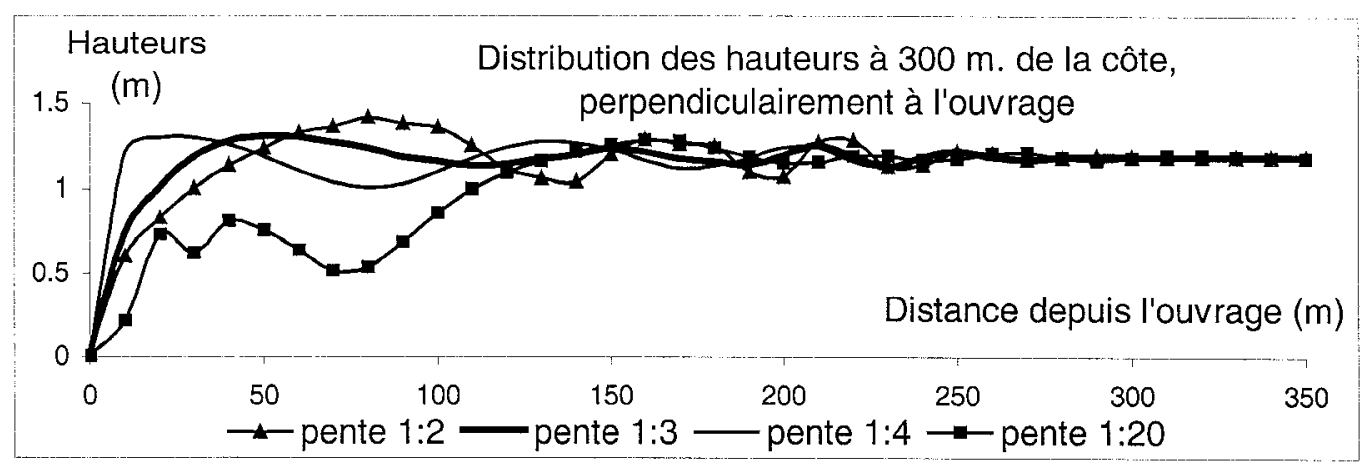

Fig. 6: Distribution des hauteurs à $300 \mathrm{~m}$ de la côte perpendiculairement à l'ouvrage pour différentes pentes.

Fig. 6: Long-shore heights distribution for different slopes. 
Quel que soit le cas de simulation, le modèle respecte les lois de réfraction et de diffraction des lignes de crêtes. En revanche, les oscillations des hauteurs sont plus ou moins importantes selon la pente modélisée.

Pour les cas-tests 1 et 2 (pentes 1:1 et 1:2), la plus forte oscillation des hauteurs est de l'ordre de $20 \%$ par rapport à la hauteur obtenue à 300 mètres de la digue. Les plages, de pente au maximum 1:20, permettent, quant à elles, la dissipation de l'énergie en front d'ouvrage. Cependant, les hauteurs sont minimisées sur une distance, perpendiculaire à la digue, de 150 mètres.

Le test réalisé avec une pente 1:3 représente le mieux la réalité (pente de la digue voisine de 1:3). De plus, on obtient une oscillation minimale des hauteurs, en l'occurrence $10 \%$. Cependant, les oscillations sont transmises sur une distance, perpendiculaire à l'ouvrage, de 200 mètres. En affinant le maillage jusqu'à obtenir des mailles de 4 mètres de côté, ce pourcentage diminue (7\%) mais les oscillations se propagent sur une distance plus importante $(500 \mathrm{~m}$.).

Par ailleurs, le site d'Anglet est exposé à des houles d'incidence variable allant jusqu'à $30^{\circ}$. Le comportement des amplitudes autour de l'ouvrage, à 300 mètres de la côte, pour les pentes $1: 2,1: 3$ et 1:4, est présenté en figure 7 .

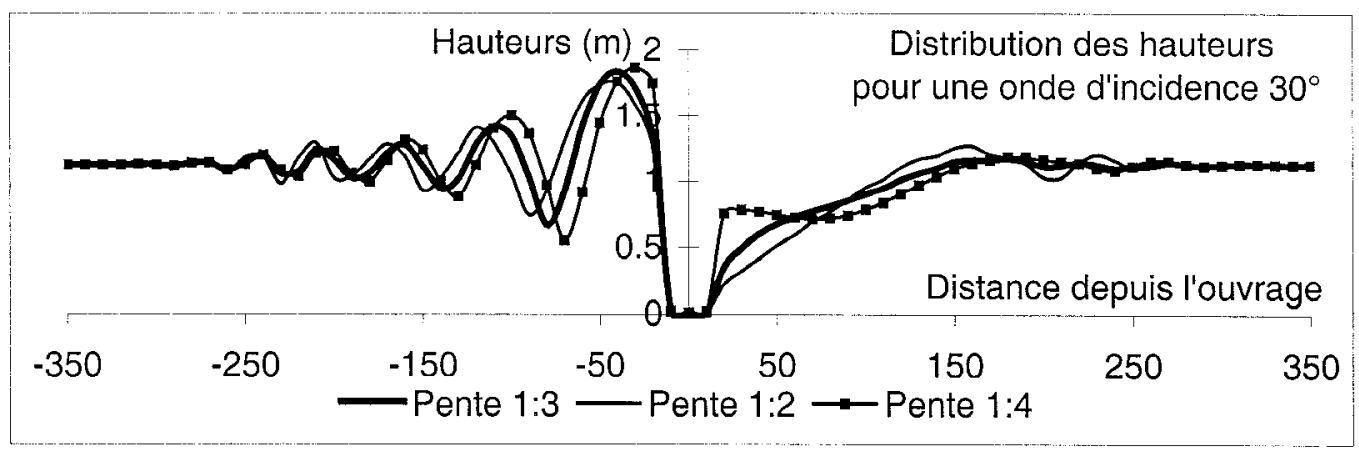

Fig. 7: Distribution des hauteurs à $300 \mathrm{~m}$ de la côte.

Fig. 7: Long-shore heights distribution.

Les oscillations des amplitudes restent importantes sur la face exposée à l'incidence de la houle. Pour une pente $1: 2$, la plus forte oscillation des hauteurs est de l'ordre de 55\% par rapport à la hauteur estimée à 350 mètres de la digue alors que pour des pentes plus douces, celle-ci atteint $65 \%$ (pente $1: 4$ ) et $62 \%$ (pente 1:3). Au contraire, sur la face opposée, la distribution des amplitudes est relativement stable pour les plages de pente maximum 1:3.

\section{Conclusion}

La connaissance des différents paramètres de houle en tous points d'un domaine d'étude est primordiale notamment pour la détermination des tensions de radiation assurant le forçage du modèle de courant (cf. figure 1). Le choix d'un modèle de propagation des ondes adapté au site étudié est indispensable. Pour des raisons d'économie de temps de calcul, le modèle REF-DIF offre un intérêt important. Résolvant l'équation de Berkhoff sous sa forme parabolique, ce modèle prend en considération les phénomènes de réfraction des ondes. Cependant, la 
résolution numérique s'effectuant ligne par ligne, aucune information provenant d'obstacles situés en aval n'est donnée (non-considération des phénomènes de réflexion) et la distribution des hauteurs met en évidence des oscillations ne représentant pas la réalité. Un système permettant de considérer les variations bathymétriques devant l'ouvrage peut limiter ces oscillations, en particulier quand l'onde est frontale devant l'ouvrage. En revanche, quand l'onde est oblique, les paramètres de houle estimés restent approximatifs dans une zone importante autour de l'obstacle modélisé. En conclusion, l'utilisation d'un modèle parabolique résolvant l'équation de Berkhoff ne semble pas appropriée à la modélisation de domaines semi-ouverts tel que le site d'Anglet.

\section{Bibliographie}

[1] De Vriend H.J., Approaches to long-term modelling of coastal morphology: a review, Coastal Engineering, 1993, vol. 21, pp 225-269.

[2] Kirby J.T., Dalrymple R.A., Documentation and user's manual REF-DIF 1 Version 2.5, CACR Report No. 94-22, 1994, 171 p.

[3] Berkhoff J.C.W., Computation combined refraction diffraction, Proc. 13 International Conference of Coastal Engineering, 1972, pp. 471-490.

[4] Radder A.C., On the parabolic equation method for water-wave propagation, Journal of Fluid Mechanics, 1979, pp. 159-176.

[5] Rapport Creocean, Amélioration des accès maritimes au port de Bayonne, Etude d'impact des travaux de rectification de l'embouchure de l'Adour, 1997, $105 \mathrm{p}$.

[6] Hallermeier R.J., Sand motion initiation by water waves: two asymptotes, Journal of Waterway, Port, Coastal and Ocean Division, ASCE, 1980, vol. 106, pp 299-318.

[7] Manoujian S., Migniot C., Etude statistique des houles entre Juillet 1972 et Juin 1973, Application au cycle de houle expérimental, Rapport L.C.H.F., 1973, $18 \mathrm{p}$.

[8] Peregrine D.H., Long waves on a beach, Journal of Fluid Mechanics, 1967, vol. 27, pp. 815-827.

[9] Wei G., Kirby J.T., A fully non-linear Boussinesq model for surface waves, Journal of Fluid Mechanics, 1995, vol. 294, pp. 71-92.

[10] Hasselmann K., Measurements of wind wave growth and swell decay during the Joint North Sea Wave Project, Deutshe Hydrographische Zeitschrift, Erganzungsheft, Reihe A, 1973. 